12

\title{
Преобразование частоты излучения мощных гиротронов в условиях обратного рамановского рассеяния на дополнительном электронном пучке
}

\author{
(С) Н.С. Гинзбург, Л.А. Юровский, И.В. Зотова, А.С. Сергеев
}

Институт прикладной физики РАН, Нижний Новгород, Россия

E-mail: ginzburg@appl.sci-nnov.ru

Поступило в Редакцию 15 ноября 2018 г.

В окончательной редакции 15 ноября 2018 г.

Принято к публикации 21 ноября 2018 г.

\begin{abstract}
Показана перспективность использования режима рамановского рассеяния попутной с электронным пучком волны накачки во встречную сигнальную волну для перестройки частоты излучения. Абсолютная неустойчивость, развивающаяся в данной системе, обеспечивает генерацию рассеянного сигнала в отсутствие внешних резонаторов, что позволяет при изменении энергии электронов плавно изменять частоту рассеянного излучения в пределах 20-40\% при использовании в качестве накачки излучения мощных гиротронов.
\end{abstract}

DOI: 10.21883/PJTF.2019.04.47329.17593

Прогресс в разработке мощных СВЧ-генераторов, в частности гиротронов [1,2], расширяет сферу перспективных приложений, для которых может быть существенно управление спектром излучения. Так, при использовании гиротронов в установках термоядерного синтеза в качестве источника для нагрева плазмы выбираются фиксированные частоты излучения, являющиеся компромиссом между поглощением в центральных и периферических областях плазмы. Вместе с тем, как показано в [3], нагрев может быть значительно эффективнее, если допустить возможность перестройки частоты падающего излучения миллиметрового диапазона. Кроме того, важным приложением сигналов с быстро перестраиваемой частотой является возможность формирования коротких импульсов с высокой пиковой интенсивностью за счет сжатия частотно-модулированного сигнала в диспергирующих линиях, например, на основе спирально гофрированных волноводов [4,5].

Для перестройки частоты в работе предлагается использовать режим вынужденного рамановского рассеяния мощного микроволнового излучения на дополнительном слаборелятивистском электронном пучке. В настоящее время рамановское рассеяние в плазме широко исследуется для компрессии и усиления импульсов оптического диапазона [6-8]. При использовании электронного пучка становятся существенными эффекты доплеровского смещения частоты, когда величина перестройки становится сравнимой по порядку с частотой накачки и рассеянного сигнала. При попутной с электронами волне накачки и встречной рассеянной волне осуществляется перекачка энергии из более высокочастотного излучения в низкочастотное с развитием абсолютной неустойчивости для рассеянного излучения. В таких условиях самовозбуждение системы имеет место в отсутствие внешних резонаторов, что обусловливает возможность плавной перестройки частоты рассеянной (сигнальной) волны при изменении энергии электронного пучка.
В качестве модели исследуем систему, изображенную на рис. 1, $a$, где попутная электронам волна накачки рассеивается в волноводе на слаборелятивистском цилиндрическом электронном пучке во встречную сигнальную волну с понижением частоты. Предполагается, что электронный пучок фокусируется сильным продольным магнитным полем $\mathbf{H}_{0}$. При рассеянии моды $\mathrm{TE}_{n, m}$ волны накачки (индекс $i$ ) в аналогичную моду рассеянного сигнала (индекс $s$ ) поля волн задаются вектор-потенциалами

$$
\mathbf{A}_{j}(\mathbf{r}, t)=\operatorname{Re}\left(\hat{A}_{j}(z) \mathbf{F}\left(\mathbf{r}_{\perp}\right) e^{i\left(\omega_{j} t-h_{j} z\right.}\right), \quad j=i, s .
$$

Здесь функции $\mathbf{F}\left(\mathbf{r}_{\perp}\right)=\varkappa_{n, m}^{-1}\left[\nabla_{\perp} \psi \times \mathbf{z}_{0}\right]$, описывающие поперечные структуры полей, выражаются через мембранные функции $\psi=J_{n}\left(\varkappa_{n, m} r_{\perp}\right) \exp (\operatorname{in} \varphi)$, где $J_{n}-$ функция Бесселя, $\varkappa_{n, m}=\sqrt{k_{j}^{2}-h_{j}^{2}}-$ поперечные волновые числа, $k_{j}=\omega_{j} / c$. Введение в систему сильного ведущего магнитного поля позволяет увеличить поперечные осцилляции электронов в поле накачки. При этом величина магнитного поля должна быть достаточно далека от циклотронного резонанса с волной накачки, а включение полей сигнала и накачки должно происходить адиабатически плавно

$$
\left(\omega_{i}-h_{i} V_{\|}-\omega_{H_{0}}\right) l_{s w} V_{\|}^{-1} \gg 2 \pi,
$$

где $l_{s w}$ - длина области включения, $\omega_{H_{0}}-$ гирочастота, $V_{\|}=\beta_{\|} c-$ поступательная скорость электронов. В противном случае будет иметь место возбуждение паразитных циклотронных осцилляций за счет отбора энергии у волны накачки.

При достаточно большой плотности электронный пучок может быть представлен как материальная среда, в которой возбуждаются волны пространственного заряда. В пучке, поглощающем энергию волны накачки, в условиях резонанса

$$
\omega_{i}-h_{i} V_{\|}-\omega_{s}-h_{s} V_{\|}=\omega_{p \|}
$$


$a$

$b$
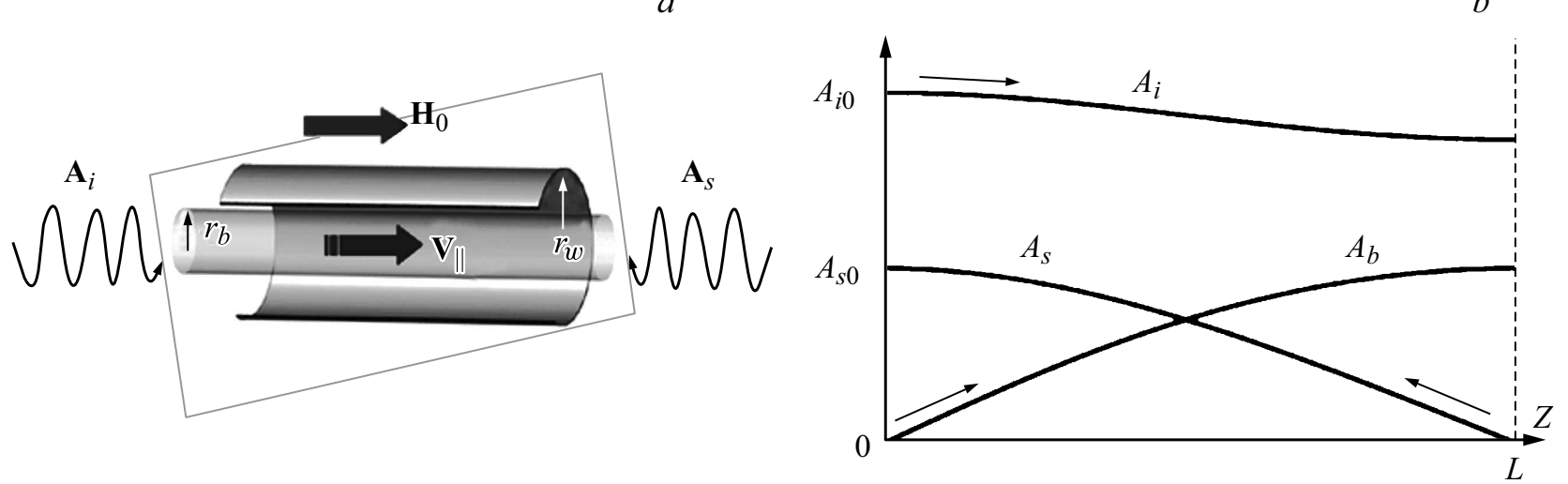

Рис. 1. $a$ - модель системы, в которой попутная волна накачки рассеивается в низкочастотную встречную волну на слаборелятивистском замагниченном электронном пучке; $b$ - продольные распределения амплитуд волн в стационарном режиме генерации, полученные из решения уравнений трехволнового взаимодействия (4) при $L=4, A_{i 0}=1$.

(где $\omega_{p \|}-$ продольная плазменная частота) возбуждается быстрая волна пространственного заряда, обладающая положительным знаком энергии. Вынужденное рамановское рассеяние на быстрой волне пространственного заряда описывается следующей системой трехволновых уравнений [9]:

$$
\frac{d A_{b}}{d Z}=A_{i} A_{s}, \quad \frac{d A_{i}}{d Z}=-A_{b} A_{s}, \quad \frac{d A_{s}}{d Z}=-A_{b} A_{i}
$$

с граничными условиями

$$
\left.A_{b}\right|_{Z=0}=0,\left.\quad A_{s}\right|_{Z=L}=0,\left.\quad A_{i}\right|_{Z=0}=A_{i 0} .
$$

Поскольку генерация имеет место при точном синхронизме (3), амплитуды волн в (4) можно считать действительными величинами [9]. Здесь $A_{i, s}=e \hat{A}_{i, s} / 2 \gamma_{0} m_{e} c^{2} \sqrt{G_{i, s}} \quad-\quad$ нормированные амплитуды волн, $A_{b}$ - амплитуда быстрой пучковой волны, $G_{i, s}=e I_{b} T / 4 \gamma_{0} m_{e} c^{2} h_{i, s} \omega_{c} r_{w}^{2} N$, $G_{b}=\operatorname{Tr}_{b} \omega_{c} c \sqrt{\mu \gamma_{0} m_{e}} / \sqrt{8 V_{\| 0} e I_{b} D}, \quad I_{b}-$ полный ток пучка, $r_{w}$ - радиус волновода, $r_{b}-$ радиус инжекции электронов, $N-$ норма волноводной моды, $Z=z \sqrt{G_{i} G_{s} G_{b}} \omega_{c} / c, L=l \sqrt{G_{i} G_{s} G_{b}} \omega_{c} / c, l-$ длина пространства рассеяния, $\mu=\beta_{\| 0}^{-2}$ - параметр группировки, $\omega_{c}=\omega_{i}-\omega_{s}-$ комбинационная частота,

$$
\begin{aligned}
D & =\left(\varkappa_{c} r_{b}\right)^{2} \frac{I_{0}\left(\varkappa_{c} r_{b}\right)}{I_{0}\left(\varkappa_{c} r_{w}\right)}\left(K_{0}\left(\varkappa_{c} r_{b}\right) I_{0}\left(\varkappa_{c} r_{w}\right)\right. \\
& \left.-I_{0}\left(\varkappa_{c} r_{b}\right) K_{0}\left(\varkappa_{c} r_{w}\right)\right)
\end{aligned}
$$

- коэффициент депрессии сил пространственного заряда, $\varkappa_{c}=\sqrt{\left(h_{i}-h_{s}\right)^{2}-\omega_{c}^{2} / c^{2}}[10]$,

$$
T=J_{n-1}^{2}\left(\varkappa_{n, m} r_{b}\right) \frac{\omega_{i}-h_{i} V_{\|}}{\omega_{i}-h_{i} V_{\|}-\omega_{H_{0}}}
$$

- коэффициент связи электромагнитных волн.

С учетом интегралов

$$
A_{i}^{2}-A_{s}^{2}=A_{i 0}^{2}-A_{s 0}^{2}, \quad A_{s}^{2}+A_{b}^{2}=A_{s 0}^{2},
$$

которые представляют собой законы сохранения числа квантов, уравнения (4) сводятся к одному уравнению для амплитуды сигнальной волны

$$
\frac{d A_{s}}{d Z}=-\sqrt{A_{i 0}^{2}-A_{s 0}^{2}+A_{s}^{2}} \sqrt{A_{s 0}^{2}-A_{s}^{2}},
$$

где $A_{s 0}=\left.A_{s}\right|_{Z=0}$ - амплитуда сигнальной волны на выходе из области рассеяния.

В стационарном режиме генерации продольное распределение амплитуд волн описывается с помощью эллиптических функций Якоби (рис. $1, b)$. Так, интегрируя (7), для амплитуды сигнальной волны имеем

$$
A_{s}=A_{s 0} \operatorname{cn}\left[A_{i 0} Z, \sqrt{R}\right] .
$$

Здесь $R=A_{s 0}^{2} / A_{i 0}^{2}-$ коэффициент использования накачки (квантовый выход), характеризующий число квантов накачки, преобразованных в кванты сигнала. Он находится из соотношения

$$
A_{i 0} L=K(\sqrt{R})
$$

которое следует из (8) при $Z=L$. Здесь $K(x)-$ полный эллиптический интеграл первого рода. При $R \rightarrow 0$ из уравнения (9) получаем условие самовозбуждения, которое для низшей продольной моды имеет вид

$$
A_{i 0} L_{s t}=\pi / 2 \text {. }
$$

Переходя к физическим переменным, для стартового тока имеем

$$
I_{s t}=2 \pi^{4} I_{0} \frac{P_{0}^{2}}{P_{i}^{2}} \frac{\omega_{i}^{2} h_{s}^{2} h_{i}^{2}}{T^{4} \omega_{c}^{4} l^{4}} \beta_{\| 0}^{7} \frac{r_{w}^{8} D N^{4}}{r_{b}^{2}},
$$

где $P_{i}-$ мощность накачки на входе в систему, $P_{0}=8.7 \mathrm{GW}, I_{0}=17 \mathrm{kA}$. Очевидно, что этот ток должен быть существенно ниже предельного вакуумного тока, пропускаемого цилиндрическим волноводом, который для замагниченного трубчатого электронного пучка 

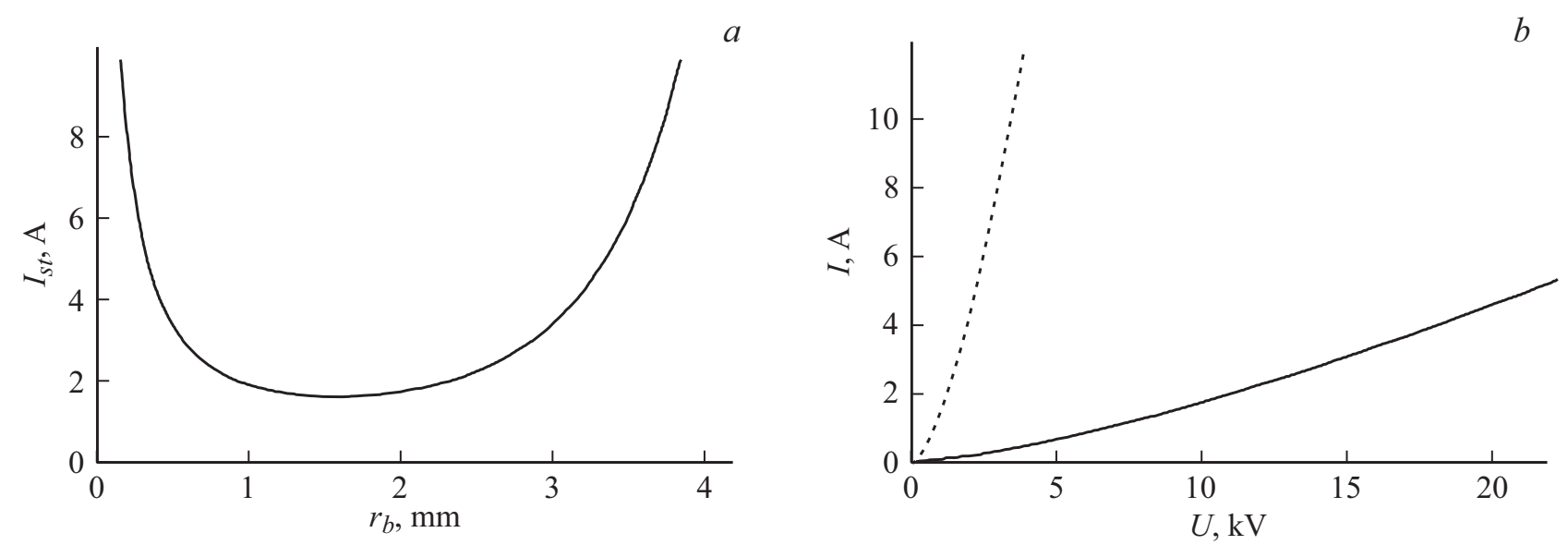

Рис. 2. $a$ - зависимость стартового тока от радиуса инжекции электронов для рассеяния мод ТЕ 1,$1 ; b-$ зависимость предельного (пунктирная кривая) и стартового (сплошная кривая) тока от ускоряющего напряжения при $r_{b}=1.6 \mathrm{~mm} . P_{i}=1 \mathrm{MW}, \lambda_{i}=2 \mathrm{~mm}$, $r_{w}=6 \mathrm{~mm}$ и $l=30 \mathrm{~cm}$.
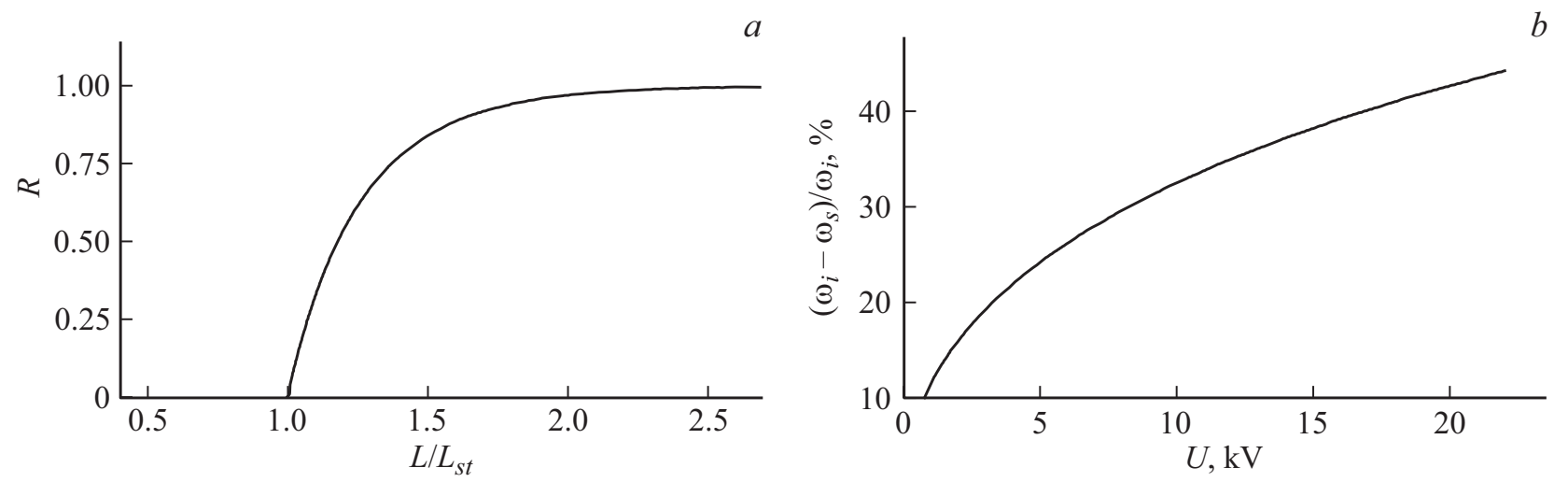

Рис. 3. $a$ - зависимость квантового выхода от длины области рассеяния; $b-$ зависимость относительной величины перестройки частоты рассеянной волны от ускоряющего напряжения. Параметры те же, что для рис. 2.

в слаборелятивистском приближении дается выражением [11]:

$$
I_{\max }=\frac{17[\mathrm{kA}]}{2 \ln \left(r_{w} / r_{b}\right)} \beta_{\| 0}^{3} .
$$

Оценки параметров проводились при мощности накачки $P_{i}=1 \mathrm{MW}$ и длине волны $\lambda_{i}=2 \mathrm{~mm}$. Предполагалось, что как волна накачки, так и рассеянная волна представляют собой моды $\mathrm{TE}_{1,1}$ цилиндрического волновода. Радиус и длина секции рассеяния были выбраны равными $r_{w}=6 \mathrm{~mm}$ и $l=30 \mathrm{~cm}$ соответственно. Величина магнитного поля $H_{0}$ была отстроена от резонансного значения на $3 \%$. Согласно рис. $2, a$, существует оптимальный радиус пучка $r_{b, \min }$, при котором достигается минимальное значение стартового тока. При выбранных параметрах он составляет $r_{b, \min }=1.6 \mathrm{~mm}$.

Дальнейшие расчеты проводились при указанном оптимальном радиусе инжекции электронного пучка. Как видно из рис. $2, b$, в том случае, когда ускоряющее напряжение находится в диапазоне значений $U=5-20 \mathrm{kV}$ величина стартового тока не превышает 6 А и остается значительно меньше предельного тока $I_{\max }$. Оценка кван- тового выхода $R$ по формуле (9) показывает, что с увеличением длины пространства рассеяния $L$ коэффициент трансформации волн асимптотически стремится к единице (рис. $3, a)$. В частности, для ускоряющего напряжения $U=10 \mathrm{kV}$, тока $I=3.5 \mathrm{~A}$ и длины области рассеяния $30 \mathrm{~cm}$ мощность сигнальной волны на выходе составляет $P_{s}=700 \mathrm{~kW}$. При увеличении тока до $I=5$ А мощность поднимается до $P_{s}=940 \mathrm{~kW}$.

Величина перестройки частоты (рис. $3, b$ ) находится из условия синхронизма (3) и при вариации напряжения в указанных пределах составляет $20-40 \%$ от частоты накачки. Заметим, что при среднем ускоряющем напряжении $10 \mathrm{kV}$ для модуляции частоты выходного излучения в пределах $\pm 3.5 \%$ от центральной частоты (например, для последующего сжатия) достаточно вариации напряжения в пределах $\pm 2.5 \mathrm{kV}$.

Как уже отмечалось, для получения генерации на комбинационном синхронизме (3) без возбуждения паразитных циклотронных колебаний необходимо адиабатически плавное включение полей, когда возбуждаются лишь вынужденные осцилляции электронов в поле накачки. Для используемого диапазона частот и энергий элек- 
тронов оценка характерной длины области включения $l_{s w}$ по формуле (2) показывает, что она находится в пределах $2.5-3 \mathrm{~cm}$. Заметим также, что напряженность радиального электрического поля на стенках волновода $E_{r}\left(r=r_{w}\right)$ указанного сечения при мегаваттном уровне мощности волны накачки составляет величину порядка $17 \mathrm{kV} / \mathrm{cm}$ и не превышает порога развития высокочастотных пробоев.

Таким образом, проведенные оценки параметров показали перспективность исследованной схемы, основанной на обратном рамановском рассеянии для плавной перестройки частоты в пределах $20-40 \%$ от частоты падающего излучения, генерируемого гиротронами мегаваттного уровня мощности. Вместе с тем очевидно, что для реализации режима вынужденного обратного рассеяния необходима высокая плотность пучков в области рассеяния. В то же время, поскольку речь идет о пучках относительно низких энергий, ток эмиссии с катода сильно ограничен (закон „трех вторых“). Однако с учетом того, что в данном случае в энергию рассеянной волны преобразуется энергия волны накачки, а не кинетическая энергия электронов, представляется перспективной схема с частичным торможением электронов перед влетом в область рассеяния. При этом анодное напряжение может быть довольно высоким, что обеспечивает достаточно высокий ток инжекции, а последующее торможение пучка позволяет обеспечить необходимую высокую плотность частиц в области рассеяния.

Работа поддержана грантом РНФ 16-42-01078.

\section{Список литературы}

[1] Belov Y.N., Chirkov A.V., Denisov G.G., Efimov E.V., Eremeev A.G., Litvak A.G., Malygin V.I., Miasnikov V.E., Popov L.G., Roy I.N., Sokolov E.V., Soluyanova E.A., Tai E.M., Usachev S.V. Completion phase of Russian gyrotron system development for ITER // Proc. of 41st Int. Conf. on infrared, millimeter, and terahertz waves. IEEE, 2016. P. 1-2. DOI: 10.1109/IRMMW-THz.2016.7758426

[2] Glyavin M.Yu., Denisov G.G. Development of high power $\mathrm{THz}$ band gyrotrons and their applications in physical research // Proc. of 42nd Int. Conf. on infrared, millimeter, and terahertz waves. IEEE, 2017. P. 1-2. DOI: 10.1109/IRMMW-THz.2017.8067024

[3] Zohm H., Thumm M. // J. Phys.: Conf. Ser. 2005. V. 25. P. 274 282.

[4] Burt G., Samsonov S.V., Phelps A.D.R., Bratman V.L., Ronald K., Denisov G.G., He W., Young A.R., Cross A.W., Konoplev I.V., Yin H., Whyte C.G. // IEEE Trans. Plasma Sci. 2005. V. 33. N 2. P. 661-667.

[5] Samsonov S.V., Phelps A.D.R., Bratman V.L., Burt G., Denisov G.G., Cross A.W., Ronald K., He W., Yin H. // Phys. Rev. Lett. 2004. V. 92. N 11. P. 118301.

[6] Malkin V.M., Shvets G., Fisch N.J. // Phys. Rev. Lett. 1999. V. 82. N 22. P. 4448-4451.

[7] Dodin I.Y., Fraiman G.M., Malkin V.M., Fisch N.J. // ЖЭТФ. 2002. T. 122. B. 4. C. 723-737.
[8] Balakin A.A., Fisch N.J., Fraiman G.M., Malkin V.M., Toroker Z. // Phys. Plasmas. 2011. V. 18. N 10. P. 102311.

[9] Гинзбург Н.С. // ЖТФ. 1985. Т. 55. В. 1. С. 47-52.

[10] Гинзбург Н.С., Новожслова Ю.В. // Радиотехника и электроника. 1984. Т. 29. № 12. С. 2419-2429.

[11] Цимринг Ш.Е. Введение в высокочастотную вакуумную электронику и физику электронных пучков. Н. Новгород: ИПФ РАН, 2012. $576 \mathrm{c.}$ 